Soren Jacobsen Grant/research support from: BMS, Christine Peschken: None declared, Diane L Kamen: None declared, Anca Askanase Consultant of: Abbvie, Grant/research support from: Glaxo Smith Kline, Astra Zeneca, Janssen, Eli Lilly and Company, Mallinckrodt, Pfizer, Bernardo Pons-Estel Consultant of: GSK, Janssen, Graciela S Alarcon: None declared.

DOI: 10.1136/annrheumdis-2021-eular.1133

\section{OP0290 1 CLINICAL CHARACTERISTICS AND PROGNOSIS OF ANTIPHOSPHOLIPID SYNDROME PATIENTS BASED ON CLUSTER ANALYSIS: A 10-YEAR COHORT STUDY}

W. Qi ${ }^{1}$, J. L. Zhao ${ }^{1}$, X. Tian ${ }^{1}$, M. LI ${ }^{1}$, X. Zeng ${ }^{1} .{ }^{1}$ Peking Union Medical College Hospital, Department of Rheumatology, Beijing, China

Background: APS is an autoimmune disease characterized by persistent antiphospholipid antibodies (aPLs) positivity, leading to thrombotic events or pregnancy morbidity. High-risk aPLs profiles included positive lupus anticoagulant (LA) and multiple aPLs positivity ${ }^{1}$. Association was also found between aPLs and a variety of manifestations beyond thrombosis, referred to "non-criteria manifestations" (i.e. thrombocytopenia, hemolytic anemia, heart valve disease and aPL-related nephropathy $)^{2}$, of which the role in APS risk stratification is poorly understood. The manifestation spectrum of APS is wide, ranging from asymptomatic aPLs positivity to life-threatening catastrophic APS, and patients other than confirmed APS also need proper management. Therefore, a risk stratification integrating demographic data, aPL-related manifestations, aPLs profiles, coexisting cardiovascular risk factors and SLE is needed for management guidance and prognosis assessment.

Objectives: Using cluster analysis, to identify phenotypes among aPL-positive patients and assess the prognosis of each phenotype.

Methods: This is a single-center, prospective cohort study of aPL-positive patients who presented to Peking Union Medical College Hospital from 2004 to 2020. Demographic characteristics, aPL-related manifestations, cardiovascular risk factors, antibodies profile and follow-up data were recorded. The primary end point was defined as a combination of newly onset arterial thrombosis (AT) or deep venous thrombosis (DVT), major bleeding events, non-criteria manifestations and all-cause death. Hierarchical cluster analysis with the Euclidean distance and the Ward method was applied to identify clusters of patients and variables separately. Multiple comparison and Kaplan-Meier survival analysis were performed among clusters.

Results: Four clusters among 383 patients (70.2\% female; mean age 37.7 years) were identified (Figure 1A). Cluster $1(n=138)$ : female patients with SLE, non-criteria manifestations, triple aPLs positivity, high AT rate and moderate DVT rate. Cluster $2(n=112)$ : male patients with obesity, smoking history, hypertension, hyperhomocysteinemia, triple aPLs positivity and the highest rate of AT and DVT. Cluster $3(n=83)$ : female patients with the highest pregnancy morbidity rate and the lowest thrombosis rate. Cluster $4(n=50): 62 \%$ male patients with isolated LA positivity, high AT rate and moderate DVT rate. Four clusters of variables were also identified (Figure 1A). From Kaplan-Meier survival analysis, 1-, 5- and 10 -year event-free survival rates were $92.6 \%, 79.8 \%$ and $66.8 \%$, respectively. Cluster 3 showed lowest incidence of primary endpoint (Figure 1B), while Cluster 1 and 2 showed higher newly-onset AT risk compared with other clusters $(P=0.028$ for 2 vs 3 and $P=0.049$ for 2 vs 4$)$.
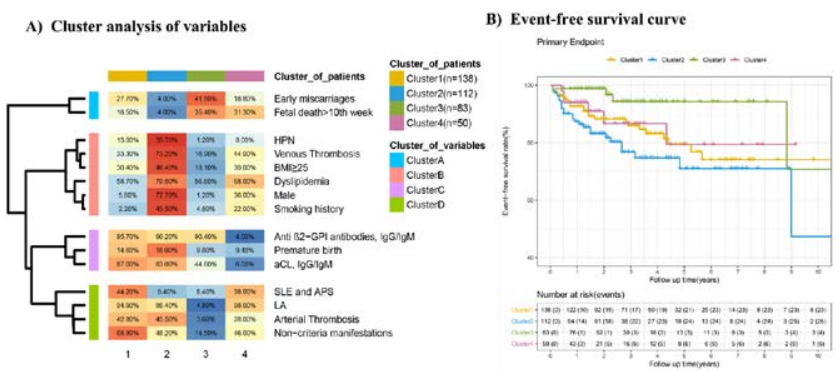

Figure 1.

Conclusion: We identified 4 clinical phenotypes of aPL-positive patients. APS secondary to SLE was always aggregated with non-criteria manifestations. Clinicians should be alert to the possibility of SLE in aPL-positive patients with coexisting non-criteria manifestations, for whom immunosuppressive therapy besides anticoagulation may be necessary. Cluster 4 represented patients with isolated LA positivity and shared similar prognosis with secondary APS and male patients, which confirmed that LA represented a high-risk antibody spectrum. Additionally, cardiovascular risk factors (i.e. male, smoking history and obesity) played an important role in thrombosis events, and led to poor prognosis.
Therefore, more attention should be paid to male patients, and the screening and management of cardiovascular risk factors should not be ignored.

\section{REFERENCES:}

[1] Tektonidou MG, Andreoli L, Limper $M$ et al. EULAR recommendations for the management of antiphospholipid syndrome in adults. Ann Rheum Dis 2019;78:1296-304

[2] Miyakis S, Lockshin MD, Atsumi T et al. International consensus statement on an update of the classification criteria for definite antiphospholipid syndrome (APS). J Thromb Haemost 2006;4:295-306.

Disclosure of Interests: None declared.

DOI: 10.1136/annrheumdis-2021-eular.1223

OP0291

\section{SEVERITY OF LABIAL MINOR SALIVARY GLAND FOCUS SCORE AND FUTURE LYMPHOMA DEVELOPMENT IN SJÖGREN'S SYNDROME}

A. Goules ${ }^{1}$, L. Chatzis ${ }^{1}$, V. Pezoulas ${ }^{2}$, C. Baldini ${ }^{3}$, F. Skopouli ${ }^{4}$

A. Venetsanopoulou ${ }^{5}$, P. Voulgari ${ }^{5}$, S. De Vita ${ }^{6}$, M. Voulgarelis ${ }^{1}, \mathrm{H}$.

M. Moutsopoulos ${ }^{7}$, D. Fotiadis ${ }^{2}$, A. Tzioufas ${ }^{1}{ }^{1}$ School of Medicine, National and Kapodistrian University of Athens, Pathophysiology, Athens, Greece; ${ }^{2}$ University of loannina, Unit of Medical Technology and Intelligent Information Systems, Athens, Greece; ${ }^{3}$ University of Pisa, Rheumatology Unit, Department of Clinical and Experimental Medicine, Athens, Greece; ${ }^{4}$ Harokopio University of Athens, Department of Nutrition and Clinical Dietetics, Athens, Greece; ${ }^{5}$ University of Ioannina, Department of Internal Medicine, Athens, Greece; ${ }^{6}$ University of Udine, Rheumatology Clinic, Department of Medical area, Athens, Greece; ${ }^{7}$ Academy of Athens, Chair Medical Sciences/Immunology, Greece, Athens, Greece

Background: The typical histologic picture of focal sialadenitis in the labial minor salivary gland (LMSG) tissues has been incorporated as a parameter in the majority of Sjögren's syndrome (SS) classification criteria, with focus score (FS) being the most widely used (1). In previous studies, higher FS has been associated with the presence of autoantibodies and extra-glandular manifestations including lymphoma, implying that FS can predict severe disease $(2,3)$. However, there are no studies exploring the association of FS with lymphoma development along with the time interval from SS diagnosis to lymphoma diagnosis.

Objectives: To investigate an association of focus score grading with lymphoma development and time to lymphoma occurrence.

Methods: From a total population 1998 consecutive patients fulfilling the 2016 ACR-EULAR criteria for SS who were followed-up in 5 Rheumatology centers from Greece and Italy (Universities of Athens, Pisa, Udine, Harokopio and Ioannina) (UPAHI group), those with positive (LMSG) (FS $\geq 1$ ) were identified. (1) Patients who had not been subjected to an LMSG biopsy or had a negative biopsy $(F S<1)$ or the biopsy was evaluated using other histologic classifications were excluded from the present study. Lymphoma patients with diagnostic LMSG biopsy performed within a year from lymphoma diagnosis as well as non-lymphoma patients with less than 1 year between SS diagnosis and their last follow up, were also excluded. Cumulative clinical and histologic data attributed to SS as defined by ESSDAI and/or ESSPRI, up to lymphoma diagnosis or last follow up, were recorded. A combined data driven Fast Correlation Based Feature selection (FCBF)/Logistic Regression (LR) model was applied on the unified dataset to identify independent lymphoma associated risk factors. A correlation between FS and time interval until lymphoma diagnosis was performed using the Pearson formula.

Results: A unified dataset of 618 SS patients with $F S \geq 1$ and at least one year of disease duration from SS diagnosis to lymphoma diagnosis or last follow up, with 30 clinical, laboratory and histologic features, was constructed. The median age at SS diagnosis was 53 (range: 15 - 80) years old, the female to male ratio was $20: 1$ and the median disease duration was 6 years (range: 1-35). Half (49\%) of the study population had focus score between one and two $(1 \leq F S<2)$, followed by a declining proportion of patients accounted for every subsequent escalating FS group. No statistically significant difference was found regarding disease duration among the various FS escalating groups $(p=0.1603$, Kruskal-Wallis test). Fifty eight patients had lymphoma with no statistically significant difference in disease duration compared to non-lymphoma controls. The data driven FCBF/LR algorithm with lymphoma on the whole dataset revealed that cryoglobulinemia $(p=0.021)$, salivary gland enlargement $(p=0.008)$ and FS $(p=0,049)$ are independent lymphoma associated risk factors. A statistically significant negative correlation was found using a Pearson formula ( $R=-0.32$ and $p=0.015)$ regarding the focus score and the time to lymphoma diagnosis.

Conclusion: This is the largest study of SS patients' histologic analysis exploring the association of LMSG FS grading with lymphoma development and the time interval until its diagnosis. Higher FS values correlate with increased lymphoma risk and early lymphoma occurrence. 